\title{
Previsão do módulo dinâmico de misturas asfálticas
}

\section{brasileiras}

\author{
Beatriz Chagas Silva Gouveia ${ }^{1}$, Jorge Barbosa Soares ${ }^{2}$, Francisco Thiago Sacramento Aragão3, \\ Luciano Pivoto Specht ${ }^{4}$
}

1Departamento Nacional de Infraestrutura de Transportes, biacsg@gmail.com

2Universidade Federal do Ceará, jsoares@det.ufc.br

3Universidade Federal do Rio de Janeiro, fthiago@coc.ufrj.br

4Universidade Federal de Santa Maria, luspecht@gmail.com

\section{Recebido:}

1 de junho de 2017

Aceito para publicação:

26 de março de 2018

Publicado:

31 de dezembro de 2018

Editor de área:

Kamilla Vasconcelos, EESC-USP

Palavras-chaves:

Misturas asfálticas;

Módulo dinâmico

Modelos de previsão.

\section{Keywords:}

Hot mix asphalt;

Dynamic modulus;

Complex modulus;

Predictive equation.

DOI:10.14295/transportes.v26i4.1388

\begin{abstract}
RESUMO
O módulo dinâmico $\left|E^{*}\right|$ é a propriedade de rigidez de misturas asfálticas mais utilizada como parâmetro de entrada no dimensionamento mecanístico-empírico de estruturas de pavimentos asfálticos. Apesar de bem estabelecido internacionalmente, ainda não há, no Brasil, normatização de ensaio para sua determinação, e ele não é considerado no método vigente de dimensionamento. Embora as metodologias de ensaio sejam bem consolidadas internacionalmente, diversos modelos de previsão de $\left|E^{*}\right|$ são reportados na literatura internacional, objetivando reduzir tempo e custos de sua obtenção experimental. No presente estudo, foram avaliados modelos de previsão de módulo dinâmico da literatura internacional, com dados de ensaios laboratoriais de 24 misturas asfálticas nacionais. Avaliados os modelos existentes, foi proposta a calibração daquele que se mostrou mais eficiente, resultando em uma equação simplificada e bem ajustada aos dados analisados. Espera-se que modelos calibrados como o aqui proposto possam ser facilitadores da aplicação, no Brasil, de métodos mecanístico-empíricos de dimensionamento de pavimentos. Esses métodos possibilitam considerar o comportamento viscoelástico dos materiais asfálticos, ou seja, levar em conta os efeitos da temperatura e da velocidade dos veículos no projeto de pavimentos.
\end{abstract}

\begin{abstract}
The dynamic modulus $\left|E^{*}\right|$ is a property of Hot-Mix Asphalt (HMA), and it is the most used stiffness parameter input in mechanistic-empirical pavement design methods. Although well established in several countries, official methodologies in Brazil for both new and rehabilitation pavement design do not consider this property. Although laboratory tests methods are internationally consolidated, several $\left|E^{*}\right|$ mastercurve predictive equations are reported on the literature, in an attempt to reduce time and costs involved on experimental testing. In this research, empirical models from the open literature to determine the $\left|E^{*}\right|$ master curve were evaluated, upon experimental data from $24 \mathrm{Bra}$ zilian mixtures. After evaluation, one calibrated equation was proposed, resulting in a simple model, and well-adjusted to the analyzed database. It is expected that calibrated models, as the one proposed in the present research, can facilitate the application, in Brazil, of mechanistic-empirical pavement design methods. These methods allow the consideration of viscoelasticity within the surface course, and therefore the effect of temperature and traffic speed in the pavement design.
\end{abstract}

\section{INTRODUÇÃO}

O conhecimento da rigidez dos materiais do revestimento e das subcamadas é necessário para possibilitar a análise da estrutura global do pavimento (tensões, deformações e deslocamentos), quando este é submetido às solicitações do tráfego e considerando os fatores ambientais. Estas rigidezes podem ser entradas como dados numéricos, quando as relações constitutivas 
assumidas são algébricas (elasticidade), ou como funções do tempo ou da frequência (viscoelasticidade). Entre os parâmetros de rigidez de misturas asfálticas adotados na literatura, o módulo dinâmico $\left(\left|E^{*}\right|\right)$ tem se destacado e sido usado com frequência em métodos mecanísticoempíricos de dimensionamento de pavimentos ou indicador de desempenho, relacionando a rigidez da mistura com a deformação permanente e a vida de fadiga observadas em campo (Witczak et al., 2002; Clyne et al., 2003; Gouveia et al., 2016).

Diversos países já desenvolveram normas próprias para a caracterização do módulo dinâmico, entre os quais destacam-se Estados Unidos, países da Comunidade Europeia, Austrália, China e Argentina. No entanto, no Brasil ainda se utiliza o ensaio de Módulo de Resiliência (DNIT, 2010), que não considera as propriedades viscoelásticas das misturas asfálticas. Specht et al. (2017) discutem esta questão, indicando restrições teóricas ao ensaio de compressão diametral, bem como apontam para a utilização do ensaio de módulo dinâmico como adequado para avaliação da rigidez de misturas asfálticas no Brasil.

Atualmente há um esforço sendo empreendido no Brasil com o objetivo de atualização do método de dimensionamento de pavimentos asfálticos em vigor, um novo método brasileiro poderá, eventualmente, considerar o módulo dinâmico como propriedade de rigidez da camada asfáltica necessária para a análise das tensões e deformações das estruturas propostas. Ainda que a comunidade de pavimentação nacional deva trabalhar para transformar esta realidade no menor espaço de tempo possível, é bem provável que o avanço desejável na caracterização de misturas asfálticas não ocorra no curto prazo. E, mesmo num momento futuro de utilização mais difundida do ensaio, é possível que nem todo projeto tenha a caracterização plenamente realizada, como projetos de menor relevância, de baixo custo, anteprojetos e estudos de viabilidade.

Objetivando reduzir o tempo e os custos, vários pesquisadores vêm tentando prever o módulo das misturas de maneira semiempírica (Bonnaure et al., 1977; Andrei et al., 1999; Christensen et al., 2003; Al-Katheeb et al., 2006; Bari e Witczak, 2006), dado que mesmo nos países que adotam $\left|E^{*}\right|$ como caracterização da rigidez de misturas asfálticas, o ensaio para sua determinação é reconhecidamente demorado, podendo custar até uma semana de trabalho para uma única mistura. Os modelos de previsão se baseiam em técnicas que relacionam a rigidez da mistura com suas características volumétricas e com as propriedades dos seus constituintes. Apesar da existência de modelos de previsão consagrados na literatura internacional, não é possível afirmar que estes modelos são aplicáveis para a previsão de módulo dinâmico de misturas brasileiras. Os modelos existentes foram criados a partir de pesquisas que se utilizaram de dados de misturas com características diferentes das misturas locais, por exemplo, origem dos ligantes, características mineralógicas dos agregados e procedimentos de dosagem distintos.

É objetivo do presente estudo avaliar a aplicabilidade de modelos de previsão de módulo dinâmico consagrados na literatura para misturas asfálticas nacionais a partir de suas características volumétricas e de propriedades dos seus constituintes. Adicionalmente, este trabalho também propõe a calibração do modelo mais eficiente entre os avaliados para fornecer uma equação preditiva para misturas brasileiras.

\section{MODELOS INVESTIGADOS}

Historicamente, os modelos preditivos com embasamento empírico têm sido desenvolvidos por meio de regressão linear ou não-linear de múltiplas variáveis a partir de dados de ensaios de laboratório e propriedades das misturas asfálticas e seus constituintes. Entre estes modelos, os 
mais usados são os propostos por Witczak e seus parceiros (Andrei et al., 1999; Bari e Witczak, 2006), cuja versão mais atual está integrada no guia norte americano de dimensionamento de pavimentos, o AASHTOWare Pavement ME Design. Outros modelos são o de Christensen et al. (2003), o de Sakhaeifar et al. (2015) e o de Mateos e Soares (2015). A seguir, são apresentadas informações sobre estes modelos, que foram os avaliados nesta pesquisa com misturas e materiais nacionais.

\subsection{Modelo de Bari e Witczak (2006)}

A primeira versão da equação de previsão de módulo dinâmico de Witczak, de 1999, considerava como variáveis alguns parâmetros volumétricos e de granulometria dos agregados da mistura asfáltica, a viscosidade do ligante e a frequência de carregamento (que se relaciona com a velocidade do tráfego). Baseou-se em uma análise de regressão multivariável de 2.750 dados experimentais obtidos de 205 misturas (Andrei et al., 1999). Em 2006, Bari e Witczak revisaram a versão de 1999 usando 7.400 dados experimentais de 346 misturas. Além de incluir 4.650 novos dados no processo de calibração da equação preditiva, Bari e Witczak (2006) propuseram uma mudança fundamental no modelo e substituíram viscosidade e frequência de carregamento pelo módulo complexo em cisalhamento do ligante, representado pelo módulo dinâmico em cisalhamento $\left(\left|\mathrm{G}_{\mathrm{b}}{ }^{*}\right|\right)$ e pelo ângulo de fase $\left(\delta_{\mathrm{b}}\right)$ na nova equação (Equação 1$)$.

$\log \left(\left|E^{*}\right|\right)=-0,349$

$$
\begin{aligned}
& +0,754\left|G_{b}{ }^{*}\right|^{-0,0052} \cdot\left(6,65-0,032 \rho_{200}+0,0027 \rho_{200}{ }^{2}+0,011 \rho_{4}-0,0001 \rho_{4}{ }^{2}+0,006 \rho_{38}\right. \\
& \left.-0,00014 \rho_{38}{ }^{2}-0,08 V_{a}-1,06 \cdot\left(\frac{V_{\text {beff }}}{V_{a}+V_{\text {beff }}}\right)\right) \\
& +\frac{\left[2,56+0,03 V_{a}+0,71 \cdot\left(\frac{V_{\text {beff }}}{V_{a}+V_{\text {beff }}}\right)+0,012 \rho_{38}-0,0001 \rho_{38}{ }^{2}-0,01 \rho_{34}\right]}{1+e^{\left(-0,7814-0,5785 \log \left|G_{b}{ }^{*}\right|+0,8834 \log \delta b\right)}}
\end{aligned}
$$

onde: $\quad\left|E^{*}\right|$ é o módulo dinâmico axial da mistura, em psi;

$\left|\mathrm{Gb}^{*}\right|$ é o módulo dinâmico em cisalhamento do ligante, em psi;

$\delta_{b}$ é o ângulo de fase do ligante, em graus;

$\rho_{200}$ é o percentual, em massa, do agregado que passa na peneira $\mathrm{n}^{0} \mathrm{200}$;

$\rho_{4}, \rho_{38}$ e $\rho_{34}$ são o percentual, em massa, do agregado retido nas peneiras $n^{\circ} 4,3 / 8$ "

e 3/4", respectivamente;

$V$ a é volume de vazios da mistura, em \% (também referido como Vv neste artigo);

$V$ beff é o volume efetivo de ligante, em \% (também referido como VBE neste artigo).

Ao final, Bari e Witczak (2006) reportaram um ajuste dos dados observados ao resultado correspondente do modelo $\left(\mathrm{R}^{2}\right)$ de $80 \%$ em escala linear e de $90 \%$ em escala logarítmica. No entanto, uma série de estudos indica que os modelos de Witczak apresentam resultados muito dispersos, especialmente nos extremos das curvas (altas e baixas temperaturas) (Schwartz, 2005; Bari e Witczak, 2006; Azari et al., 2007; Ceylan et al., 2009).

\subsection{Modelo de Christensen et al. (2003)}

O modelo semiempírico de Christensen et al. (2003) é baseado na lei das misturas desenvolvida por Y. J. Hirsch na década de 1960 e também tem sido bastante usado por pesquisadores e técnicos. A equação preditiva do modelo é simples e inclui parâmetros volumétricos da mistura, 
rigidez do ligante e volume de contato do agregado (Pc), um parâmetro introduzido pelos autores e calculado a partir destes mesmos parâmetros da equação preditiva. 0 modelo foi desenvolvido a partir de 206 dados de 18 misturas, considerado pelos autores um volume razoável de dados para o desenvolvimento inicial. A expressão final para a versão simplificada do modelo é apresentada nas Equações 2 e 3. Christensen et al. (2003) reportaram um ajuste dos dados observados ao resultado correspondente do modelo $\left(\mathrm{R}^{2}\right)$ de $96,8 \%$.

$$
\begin{gathered}
\left|E^{*}\right|=P_{C}\left[4.200 .000\left(1-\frac{V M A}{100}\right)+3\left|G_{b}{ }^{*}\right|\left(\frac{V F A . V M A}{10.000}\right)\right]+\left(1-P_{c}\right)\left[\frac{1-\frac{V M A}{100}}{4.200 .000}+\frac{V M A}{3 . V F A \cdot\left|G_{b}{ }^{*}\right|}\right]^{-1} \\
P_{c}=\frac{\left(20+\frac{V F A .3\left|G_{b}{ }^{*}\right|}{V M A}\right)^{0,58}}{650+\left(\frac{V F A .3\left|G_{b}{ }^{*}\right|}{V M A}\right)^{0,58}}
\end{gathered}
$$

onde: $\quad\left|E^{*}\right|$ é o módulo dinâmico da mistura, em psi;

$\left|G_{b}{ }^{*}\right|$ é o módulo dinâmico em cisalhamento do ligante, em psi;

$P_{c}$ é volume de contato do agregado;

$V M A$ é o vazio do agregado mineral, em \%;

$V F A$ é o vazio preenchido com asfalto, em \%.

\subsection{Modelo de Sakhaeifar et al. (2015)}

Em estudo realizado na Carolina do Norte (Sakhaeifar et al., 2015) foram desenvolvidos modelos de regressão para previsão de $\left|E^{*}\right|$ levando em consideração os conceitos de viscoelasticidade e diferentes propriedades físicas e mecânicas de ligantes e misturas. Os pesquisadores contaram com uma base de 1.008 misturas. Ao final, os autores apresentaram 2 modelos, denominados de global, mais complexo, e global simplificado. 0 modelo global simplificado (Equação 4), utilizado no presente estudo, apresentou $R^{2}=0,96$ na escala aritmética e $R^{2}=0,98$ na escala logarítmica para os dados primários utilizados na pesquisa.

$$
\begin{aligned}
& \log \left(\left|E^{*}\right|\right)=6,4197-0,00014 \rho_{34}{ }^{2}-0,00547 \rho_{38}-0,11786 \rho_{200}-0,05528 V_{a}-0,16266 V_{\text {beff }}+ \\
& 0,00487 V_{\text {beff }}{ }^{2}+\frac{\left[0,57677+0,00713 \rho_{38}+0,16167 \rho_{200}-0,0052 \rho_{200}{ }^{2}+0,01889 V_{a}+0,16031 V_{\text {beff }}-0,00592 V_{\text {beff }}{ }^{2}\right]}{1+e^{\left(1,8645-0,95991 \log \left|G_{b}{ }^{*}\right|\right)}}
\end{aligned}
$$

onde: $\quad\left|E^{*}\right|$ é o módulo dinâmico axial da mistura, em psi;

$\left|G_{b}{ }^{*}\right|$ é o módulo dinâmico em cisalhamento do ligante, em psi;

$\rho_{200}$ é o percentual, em massa, do agregado que passa na peneira $\mathrm{n}$ o 200 ;

$\rho_{38}$ e $\rho_{34}$ são o percentual, em massa, do agregado retido nas peneiras $3 / 8$ " e $3 / 4$ ",

respectivamente;

$V_{a}$ é volume de vazios da mistura, em \% (também referido como Vv neste artigo);

Vbeff é o volume efetivo de ligante, em \% (também referido como VBE neste artigo).

\subsection{Modelo de Mateos e Soares (2015)}

Mateos e Soares (2015) propuseram uma equação simplificada para determinação de |E*|. 0 modelo foi calibrado a partir de dados experimentais de 8 misturas asfálticas espanholas. A Equação 5 apresenta a função sigmoidal do modelo proposto, que apresentou um ajuste aos dados experimentais com $\mathrm{R}^{2}$ de $98,7 \%$ em escala logarítmica.

$$
\log \left(\left|E^{*}\right|\right)=1,572+\frac{2,894}{1+e^{(-1,495+10,173 V v-0,5837 \log (|G *|))}}
$$


onde: $\quad\left|G^{*}\right|$ é o módulo dinâmico em cisalhamento do ligante, em $\mathrm{MPa}$;

Vv é o percentual de vazios da mistura.

\subsection{Outras Considerações}

Para utilização dos modelos de previsão em misturas de características diversas daquelas utilizadas na geração das equações, os modelos devem ser calibrados localmente, já que seu desempenho varia conforme as características dos materiais. As calibrações locais contribuem para uma previsão mais acurada do módulo dinâmico, levando em conta as características específicas das misturas e seus constituintes tais como origem, constituição e especificação dos ligantes, origem e características dos agregados e parâmetros próprios de dosagem.

Em estudo abrangente Schwartz (2005) indica que diferenças do valor do módulo medido e previsto apresentam sensibilidade muito pequena no dimensionamento da espessura da camada asfáltica de um pavimento (Underwood et al., 2011). No entanto, quanto à previsão de desempenho da mistura asfáltica, esta se mostrou sensível à diferença do valor do módulo medido e calculado. Kim et al. (2005) relataram que diferenças de 50\% entre módulos medidos e previstos acarretam erros de 25 a 50\% na previsão da vida de fadiga e erros de até $80 \%$ na previsão do desempenho quanto à deformação permanente. Já o dimensionamento da estrutura do pavimento, que considera, além da rigidez da camada asfáltica, as características das subcamadas, e os efeitos do clima, do tráfego e de outros fatores de campo, foi observada uma sensibilidade muito pequena, mesmo com grande variação entre os valores dos módulos medidos e calculados.

\section{ESTUDO EXPERIMENTAL}

\subsection{Materiais}

Um total de 24 misturas oriundas de diferentes regiões do país foi utilizado como fonte de dados para este estudo. Todos os detalhes das misturas estudadas estão em Oliveira (2014), Mattos (2014), Freire (2015), Almeida Júnior (2016) e Gouveia (2016). A Tabela 1 apresenta as principais variáveis qualitativas das misturas que compõem a investigação.

Tabela 1: Quantidade de misturas por variável qualitativa

\begin{tabular}{lll}
\hline Variável & Tipo & Quantidade \\
\hline \multirow{3}{*}{ Ligante } & $50 / 70$ & 13 \\
& $50 / 70+$ Fresado & 1 \\
& $30 / 45$ & 1 \\
& $60 / 85+$ Polímero & 9 \\
\hline \multirow{2}{*}{ Faixa granulométrica } & Faixa B DNIT & 11 \\
& Faixa C DNIT & 12 \\
\hline \multirow{2}{*}{ Método de dosagem } & Faixa III DERSA & 1 \\
\hline \multirow{3}{*}{ Teor de ligante $(\mathrm{Pb})$} & Marshall & 11 \\
& Superpave & 13 \\
\hline Volume de vazios do corpo de & Pb $<4,0 \%$ & 5 \\
prova & $4,0 \% \geq$ Pb $>5,0 \%$ & 16 \\
\hline \multirow{2}{*}{ Estado de origem } & Pb $\geq 5,0 \%$ & 3 \\
\hline Aplicação & $4,0 \%$ & 8 \\
& CE & 16 \\
\hline
\end{tabular}




\subsection{Ensaios de Laboratório}

Os ensaios de determinação de $\left|\mathrm{E}^{*}\right|$ das misturas asfálticas foram realizados seguindo as recomendações da norma norte americana AASHTO T 342-11. No entanto, o processo de fabricação recomendado pela norma, em que o núcleo de $100 \mathrm{~mm}$ é extraído de uma amostra compactada no compactador giratório Superpave de $150 \mathrm{~mm}$, foi seguido somente para o corpo de prova da mistura oriunda de São Paulo. Para as demais misturas, não foi possível a extração dos núcleos dos corpos de prova, que foram moldados no diâmetro final requerido para o ensaio. Cabe ressaltar que estudo de Robinette e Williams (2006) mostra que a metodologia de preparação dos corpos de prova, com ou sem extração do núcleo, não afeta a resposta do material no ensaio de módulo dinâmico por compressão axial.

Dado que as propriedades reológicas dos ligantes a serem utilizadas nos modelos de previsão devem refletir a condição real de envelhecimento da mistura (Mateos e Soares, 2015), as amostras de cada ligante foram ensaiadas em reômetro de cisalhamento dinâmico, após serem submetidas ao envelhecimento no Rolling Thin Film Oven Test - RTFOT, visando à obtenção das propriedades reológicas dos ligantes, quais sejam, as curvas do módulo dinâmico de cisalhamento e do ângulo de fase, correspondentes às misturas pós-compactação. 0 ensaio foi realizado em uma amostra de cada ligante, utilizando a geometria de placas paralelas de $8 \mathrm{~mm}$ de diâmetro, conforme AASHTO T 315-12. Para cada ligante, foi feita uma varredura de frequências, a deformação controlada, com amplitude variando entre 0,4\% e 0,5\%. Este intervalo foi determinado a partir de resultados de testes de varredura de deformação, que foram previamente realizados para a definição dos limites de linearidade do comportamento reológico dos ligantes.

0 intervalo de frequências adotado nas varreduras foi de $0,1 \mathrm{~Hz}$ a $25 \mathrm{~Hz}$. As temperaturas testadas foram as mesmas do ensaio de $\left|\mathrm{E}^{*}\right|$ das misturas $\left(54,4 ; 37,8 ; 21,1 ;\right.$ e $4,4^{\circ} \mathrm{C}$, nesta ordem), exceto $\mathrm{a}-10^{\circ} \mathrm{C}$, por restrições do equipamento, além de estar fora da zona de temperaturas encontradas nos pavimento brasileiros. A varredura de frequências foi realizada sempre da frequência mais baixa para a mais alta, conforme AASHTO T 315-06 para a geometria de $8 \mathrm{~mm}$, com intervalo mínimo de 10 minutos para o equilíbrio de cada temperatura.

\subsection{Avaliação dos Resultados}

O desempenho dos modelos foi avaliado pela comparação dos valores de módulo medidos em laboratório com os valores calculados por meio dos modelos de previsão. Isto foi possível ao se plotarem os valores de módulos medidos e previstos em um mesmo gráfico. 0 ajuste dos pontos em relação à linha de igualdade foi determinado pelos parâmetros estatísticos $\mathrm{R}^{2}$ e $\mathrm{Se} / \mathrm{Sy}$, onde $\mathrm{R}^{2}$ é o coeficiente de correlação entre os valores medidos e estimados de $\left|E^{*}\right|$, e Se/Sy é a razão entre o erro padrão dos valores estimados de $\left|E^{*}\right|$ e o desvio padrão dos valores medidos de |E*| (Witczak et al., 2002; Robbins, 2009; El-Badawy et al., 2012; Mohamad et al., 2014). A razão Se/Sy reflete a melhoria na acurácia da previsão de uma equação preditiva. Valores menores (mais próximos de 0) indicam previsões melhores. Os valores de $\mathrm{R}^{2}$, Se e Sy podem ser determinados por meio das Equações 6, 7, e 8.

$$
\begin{gathered}
R^{2}=1-\frac{(n-k)}{(n-1)}\left(\frac{S e}{s y}\right)^{2} \\
S e=\sqrt{\frac{\sum(y i-\hat{y} i)^{2}}{(n-k)}} \\
S y=\sqrt{\frac{\sum(y i-\bar{y})^{2}}{(n-1)}}
\end{gathered}
$$


onde: $\quad$ yi é cada módulo dinâmico axial medido;

ŷi é cada módulo dinâmico axial previsto;

y é a média do módulo dinâmico axial medido;

n é o tamanho da amostra;

k é o número de variáveis independentes do modelo investigado.

Witczak et al. (2002) propuseram os critérios mostrados na Tabela 2 para a avaliação dos parâmetros estatísticos de ajuste dos dados. Estes critérios foram adotados neste trabalho.

Tabela 2: Classificação do ajuste de dados, adaptado de Witczak et al. (2002)

\begin{tabular}{lll}
\hline Critério & $\mathbf{R}^{\mathbf{2}}$ & Se/Sy \\
\hline Excelente & $>0,90$ & $<0,35$ \\
Bom & $0,70-0,89$ & $0,36-0,55$ \\
Razoável & $0,40-0,69$ & $0,56-0,75$ \\
Ruim & $0,20-0,39$ & $0,76-0,90$ \\
Muito Ruim & $<0,19$ & $>0,90$ \\
\hline
\end{tabular}

Uma segunda análise realizada se refere à inclinação e ao intercepto da linha de regressão linear dos pontos plotados no gráfico $\left|\mathrm{E}^{*}\right|$ experimental versus $\left|\mathrm{E}^{*}\right|$ previsto, em relação à linha de igualdade, conforme realizado no estudo de El-Badawy et al. (2012). A equação de regressão linear dos dados experimentais em um ajuste ideal deverá apresentar inclinação próxima de 1 e intercepto próximo de 0 .

\section{ANÁLISE DOS DADOS E RESULTADOS}

\subsection{Reologia dos Ligantes}

O módulo dinâmico de cisalhamento $\left(\left|G^{*}\right|\right)$ do ligante é um input comum em todas as equações investigadas de previsão de $\left|\mathrm{E}^{*}\right|$ da mistura correspondente. Assim, a Figura 1 apresenta as curvas mestras experimentais de $\left|\mathrm{G}^{*}\right|$ e também da outra propriedade reológica no domínio da frequência, o ângulo de fase $\varphi$, dos ligantes utilizados nas misturas. A temperatura de referência das curvas é de $20^{\circ} \mathrm{C}$.
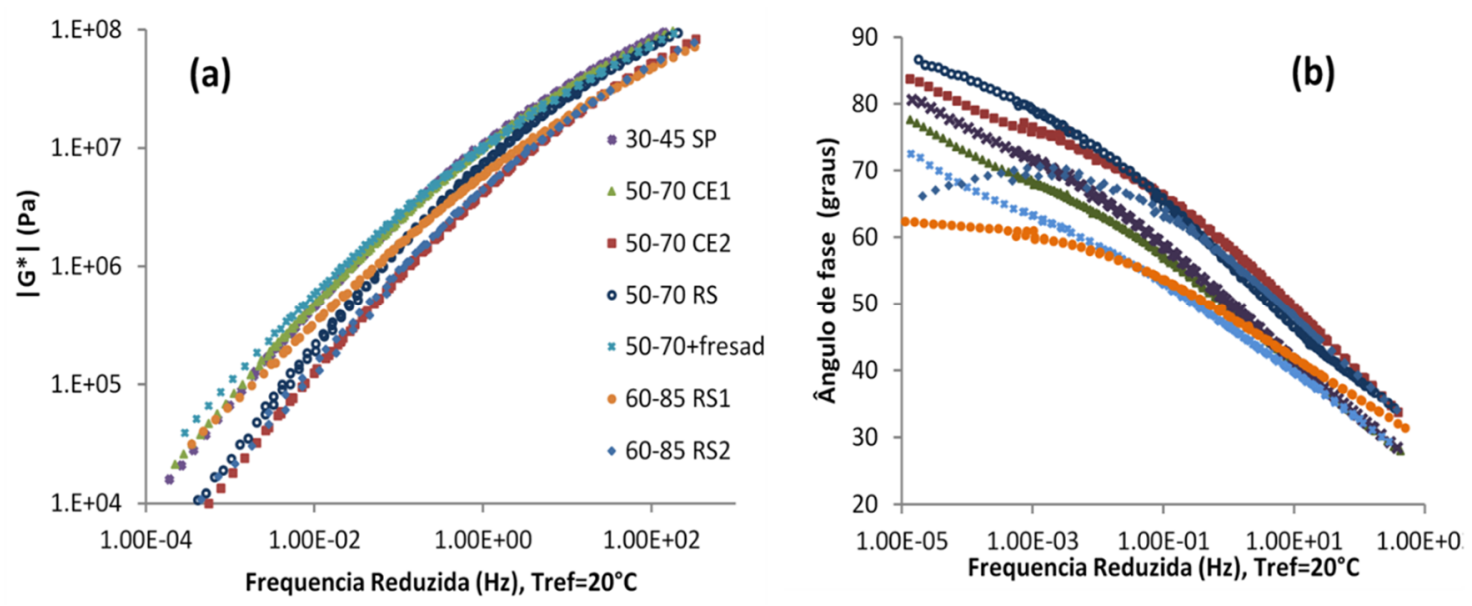

Figura 1: Propriedades reológicas dos ligantes investigados

Os dados apontam que os ligantes mais consistentes (CAP 30/45SP e CAP50/70+fresado), apresentaram rigidezes superiores aos demais ligantes. Cabe destacar, ainda, que o CAP 
50/70CE1 apresentou curva de rigidez semelhante ao CAP 30/45SP, e muito distinta do CAP 50/70CE2, indicando que a classificação por penetração vigente no Brasil não necessariamente reflete as propriedades reológicas dos ligantes. Já as curvas dos ângulos de fase dos ligantes modificados apresentaram uma maior suavização e uma redução na região de temperaturas mais altas, quando comparadas às curvas correspondentes aos ligantes puros, indicando que os mesmos apresentam um comportamento mais elástico.

\subsection{Modelos de Previsão de Módulo Dinâmico}

\subsubsection{Modelo de Bari e Witczak (2006)}

A Figura 2 apresenta os resultados da previsão das curvas de $\left|E^{*}\right|$ das misturas pela equação de Bari e Witczak (2006) aplicada para o conjunto dos dados primários e secundários. Para o conjunto de 709 pontos de dados utilizados na análise, o modelo apresentou um $\mathrm{R}^{2}$ de 0,82 e Se/Sy de 0,79 em escala logarítmica e $\mathrm{R}^{2}$ de 0,59 e Se/Sy de 0,79 em escala linear. Os dados na Figura 2 indicam que a equação Witczak tende a superestimar os valores de $\left|E^{*}\right|$, especialmente nas temperaturas mais altas. Esse resultado corrobora os resultados reportados por Azari et al. (2007), Robbins (2009), Mateos e Soares (2015).

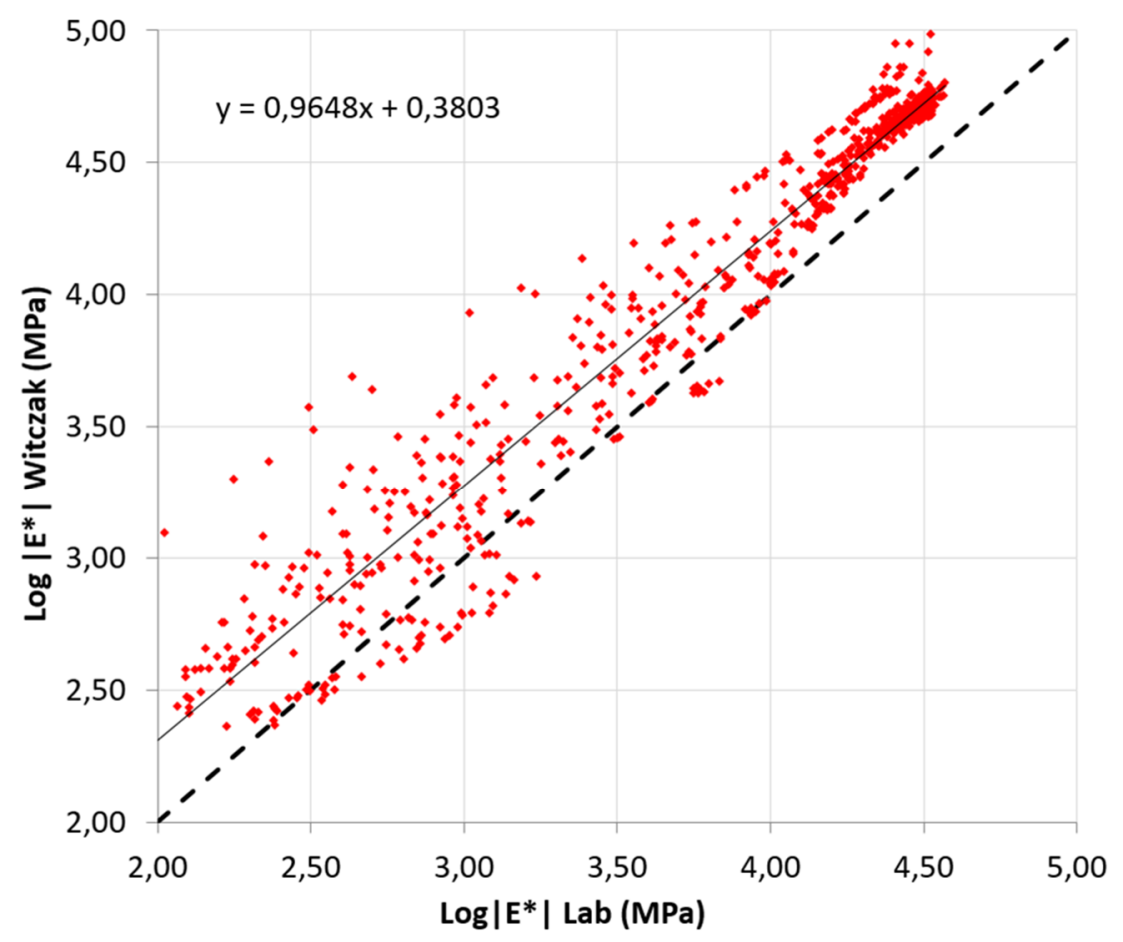

Figura 2: $\log \left(\left|E^{*}\right|\right)$ medido vs $\log \left(\left|E^{*}\right|\right)$ Witczak, para o conjunto de dados

\subsubsection{Modelo de Christensen et al. (2003)}

A Figura 3 apresenta os resultados da previsão das curvas de $\left|E^{*}\right|$ das misturas pela equação de Christensen et al. (2003) aplicada para um conjunto de 576 dados. Este modelo gerou uma inversão na tendência dos valores calculados de $\left|E^{*}\right|$ quando o $\left|G^{*}\right|>1$ psi, sendo que estes foram descartados da análise. Recomenda-se, assim, que a utilização do modelo se limite a $\left|G^{*}\right|>1$ psi. Para os 576 pontos de dados, o modelo apresentou um $\mathrm{R}^{2}$ de 0,73 e Se/Sy de 0,52 em escala logarítmica e $\mathrm{R}^{2}$ de 0,63 e Se/Sy de 0,61 em escala linear. 


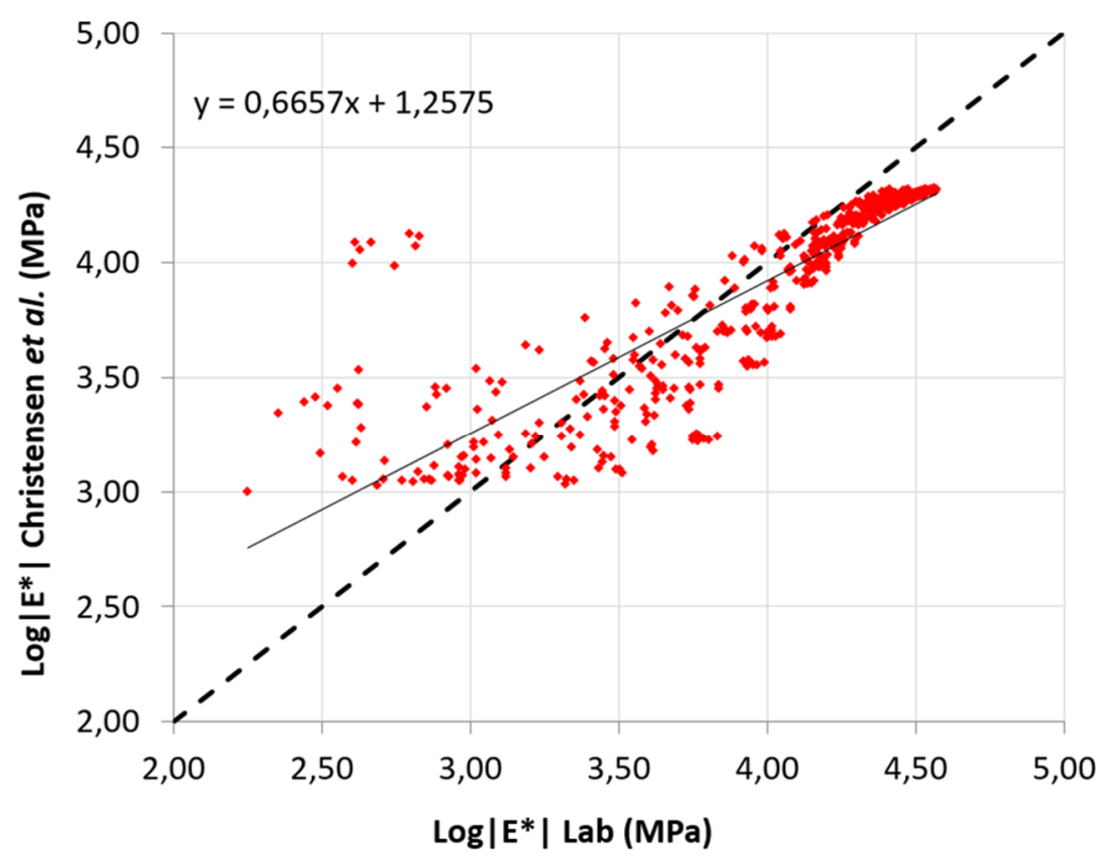

Figura 3: $\log \left(\left|\mathrm{E}^{*}\right|\right)$ medido vs $\log \left(\left|\mathrm{E}^{*}\right|\right)$ Christensen et al., para o conjunto de dados

\subsubsection{Modelo de Sakhaeifar et al. (2015)}

A Figura 4 apresenta os resultados da previsão das curvas de $\left|E^{*}\right|$ das misturas pela equação simplificada proposta por Sakhaeifar et al. (2015) aplicada para o conjunto dos dados. Para os 720 pontos de dados, o modelo apresentou um $\mathrm{R}^{2}$ de 0,91 e Se/Sy de 0,31 em escala logarítmica e $\mathrm{R}^{2}$ de 0,94 e Se/Sy de 0,24 em escala linear. Sakhaeifar et al. (2015) relataram um $\mathrm{R}^{2}$ de 0,96 em escala linear e de 0,98 em escala logarítmica para os dados que deram origem à equação simplificada.

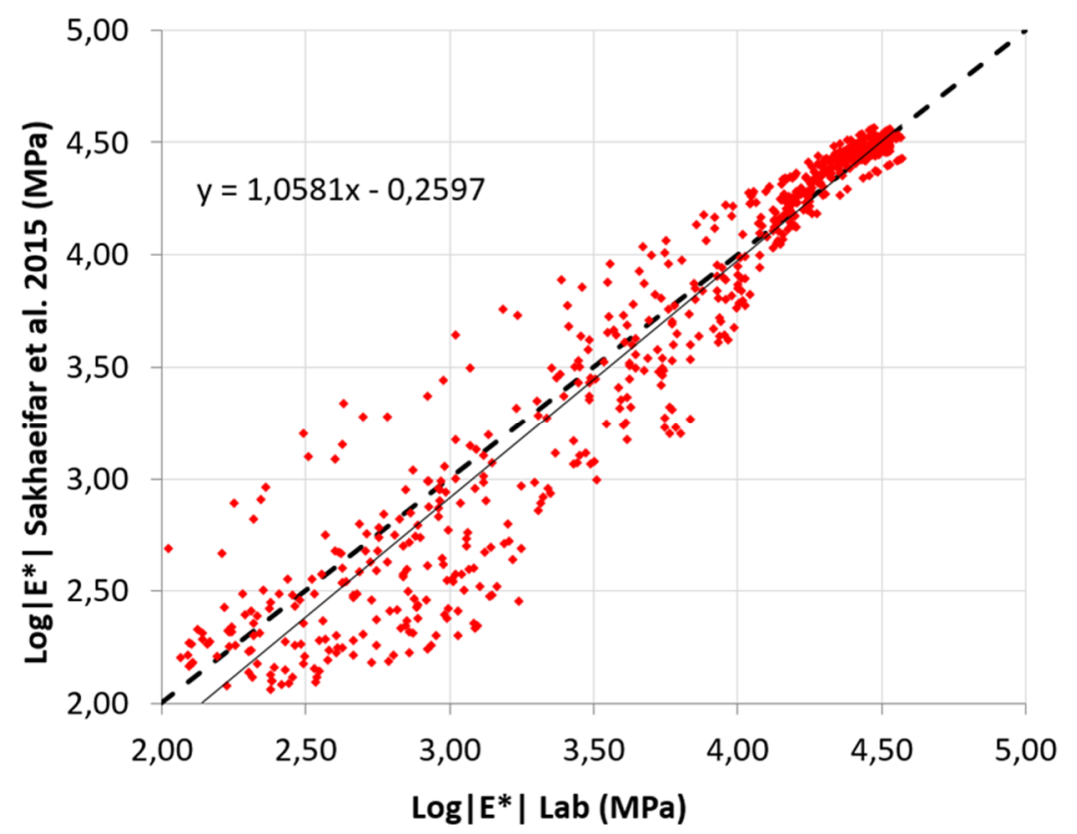

Figura 4: $\log \left(\left|E^{*}\right|\right)$ medido vs $\log \left(\left|E^{*}\right|\right)$ Sakhaeifar et al., para o conjunto de dados 


\subsubsection{Modelo de Mateos e Soares (2015)}

A Figura 5 apresenta os resultados da previsão das curvas de $\left|E^{*}\right|$ das misturas pela equação simplificada proposta por Mateos e Soares (2015) aplicada para o conjunto dos dados. Para os 720 pontos de dados, o modelo apresentou um $\mathrm{R}^{2}$ de 0,90 e Se/Sy de 0,43 em escala logarítmica e $\mathrm{R}^{2}$ de 0,94 e Se/Sy de 0,34 em escala linear. Mateos e Soares (2015) relataram um $\mathrm{R}^{2}$ de 0,96 em escala linear e de 0,99 em escala logarítmica para os dados que deram origem à equação.

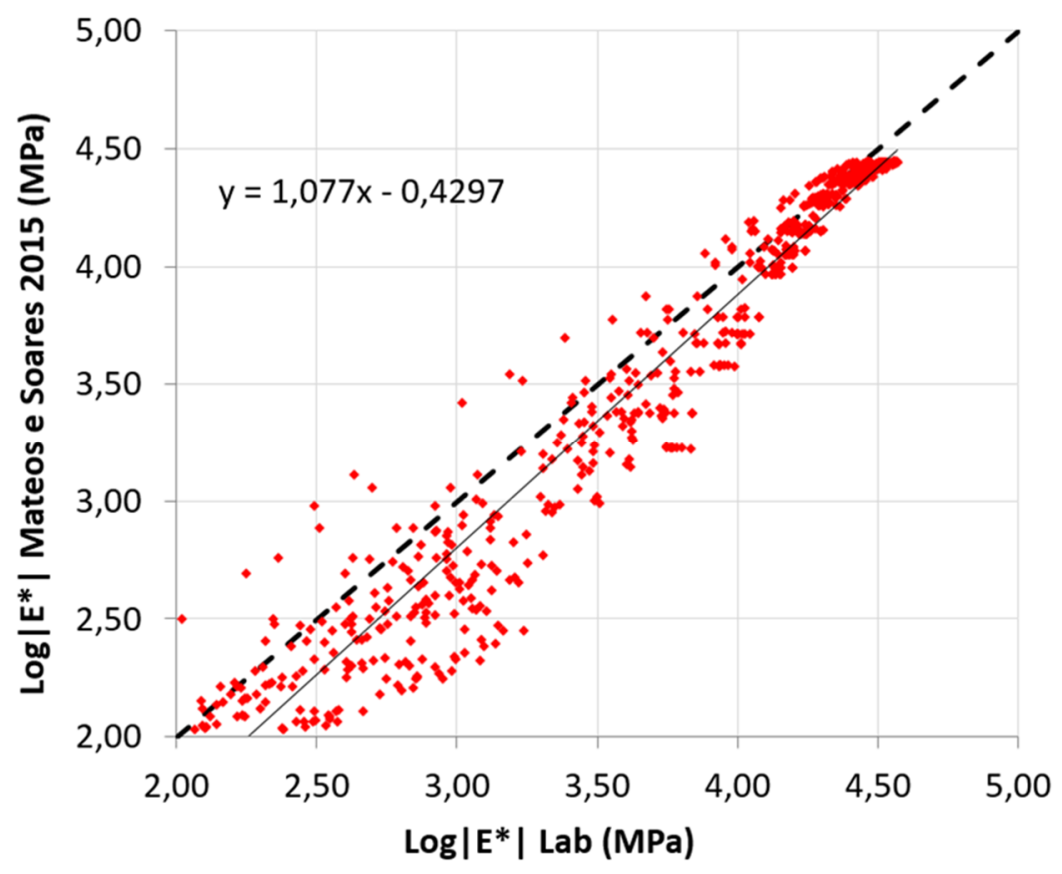

Figura 5: $\log \left(\left|E^{*}\right|\right)$ medido vs $\log \left(\left|E^{*}\right|\right)$ Mateos e Soares, para o conjunto de dados

\subsubsection{Comparativo entre os Modelos}

A Tabela 3 apresenta os indicadores estatísticos de ajuste dos dados experimentais aos dados modelados, em escala linear e em escala logarítmica, considerando a conceituação de desempenho proposta na Tabela 2.

Tabela 3: Indicadores estatísticos em escala linear e logarítmica

\begin{tabular}{l|llll|lll}
\hline \multirow{2}{*}{ Modelo } & \multicolumn{4}{l|}{ Escala Linear } & \multicolumn{3}{l}{ Escala Logarítmica } \\
\cline { 2 - 7 } & $\mathbf{R}^{\mathbf{2}}$ & Se/Sy & Desempenho & $\mathbf{R}^{\mathbf{2}}$ & Se/Sy & Desempenho \\
\hline Bari e Witczak (2006) & 0,59 & 0,79 & Razoável & 0,82 & 0,42 & Bom \\
Christensen et al. (2003) & 0,63 & 0,61 & Razoável & 0,73 & 0,52 & Bom \\
Sakhaeifar et al. (2015) & 0,94 & 0,24 & Excelente & 0,91 & 0,31 & Excelente \\
Mateos e Soares (2015) & 0,94 & 0,34 & Excelente & 0,90 & 0,43 & Excelente \\
\hline
\end{tabular}

Uma análise do intercepto e da inclinação das retas de regressão das nuvens de pontos dos modelos apresentadas nos gráficos acima também reflete o desempenho dos modelos. A Tabela 4 apresenta os resultados dessa avaliação, sendo que uma inclinação mais próxima de 1 e um intercepto mais próximo de 0 indicam um melhor ajuste dos pontos à linha de igualdade.

A investigação dos modelos indica que as equações propostas por Mateos e Soares (2015) e Sakhaeifar et al. (2015) apresentaram os melhores ajustes dos dados, vide Tabelas 3 e 4 . Ambos 
apresentaram excelente coeficiente de correlação entre os dados medidos e estimados. Em ambos os modelos, a reta de regressão da nuvem de pontos se aproximou mais da linha de igualdade, indicando que um pequeno ajuste nos coeficientes é capaz de otimizar a previsão dos valores dos módulos. A nuvem de pontos gerada pelo modelo de Bari e Witczak (2006) indica que a equação tende a superestimar os valores dos módulos, embora a reta de regressão apresente uma inclinação bem próxima de 1. Já o modelo de Christensen et al. (2003) apresentou o pior desempenho, com uma forte tendência de superestimar os valores dos módulos em altas temperaturas/baixas frequências.

Tabela 4: Avaliação das retas de regressão

\begin{tabular}{l|ll}
\hline Modelo & Inclinação & Intercepto \\
\hline Bari e Witczak (2006) & 0,96 & 0,38 \\
Christensen et al. (2003) & 0,67 & 1,26 \\
Sakhaeifar et al. (2015) & 1,06 & 0,26 \\
Mateos e Soares (2015) & 1,08 & 0,43 \\
\hline
\end{tabular}

\section{PROPOSTA DE CALIBRAÇÃO DA EQUAÇÃO DE MATEOS E SOARES (2015)}

Por ser mais simples, dado o número reduzido de parâmetros, e por apresentar forte correlação entre os dados previstos e os obtidos em laboratório, a equação de Mateos e Soares (2015) foi avaliada como um modelo de grande potencial. Assim, foi proposto um refinamento da calibração deste modelo (Equação 9) a partir de todos os 720 dados disponíveis, resultando em um $\mathrm{R}^{2}$ de 0,96 e um Se/Sy de 0,23 em escala logarítmica e $\mathrm{R}^{2}$ de 0,97 e Se/Sy de 0,23 em escala linear. Conforme os critérios apresentados na Tabela 2, a equação calibrada pode ser classificada como excelente.

$$
\log \left(\left|E^{*}\right|\right)=2,259+\frac{2,423}{1+e^{(1,274-28,685 V v-0,829 \log (\mid G *))}}
$$

onde: $\quad\left|G^{*}\right|$ é o módulo dinâmico em cisalhamento do ligante na mesma frequência reduzida da mistura, MPa;

$V v$ é o percentual de vazios da mistura.

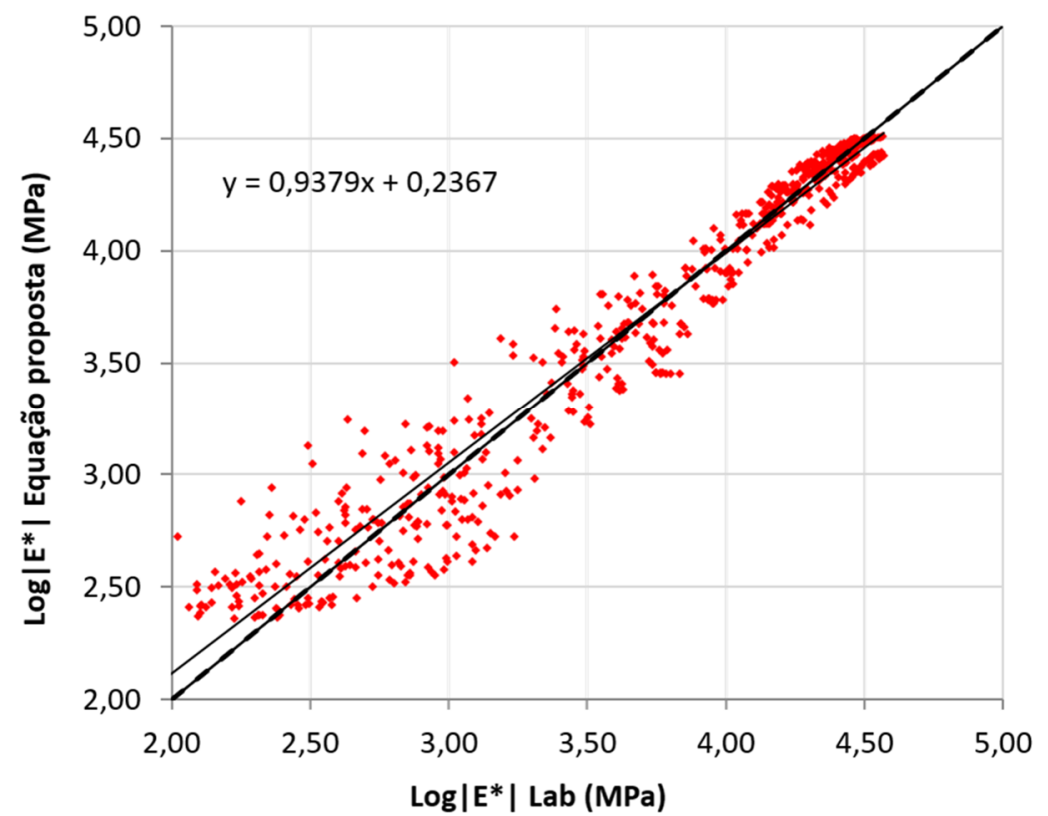

Figura 6: Ajuste dos dados de calibração do modelo de Mateos e Soares (720 pontos) 
Tabela 5: Avaliação das retas de regressão da calibração proposta

\begin{tabular}{lll}
\hline Modelo & Inclinação & Intercepto \\
\hline Mateos e Soares (2015) & 1,08 & 0,43 \\
Calibração proposta & 0,94 & 0,24 \\
\hline
\end{tabular}

A Figura 6 mostra a relação entre os módulos dinâmicos previstos e obtidos em laboratório, em escala logarítmica. Como observado, a nuvem de dados é concentrada em uma região próxima da linha de igualdade, o que indica a forte correlação entre os dois grupos de dados. Esta forte correlação é confirmada a partir da análise da reta de regressão da nuvem de pontos, cujos resultados são apresentados na Tabela 5. Observa-se que a inclinação da reta de regressão se aproximou de 1, e o intercepto se aproximou de zero.

\subsection{Incremento do Banco de Dados}

A calibração continuada da equação proposta a partir do incremento do banco de dados existente é possível e desejável. Para tanto, inicialmente é interessante a criação de um banco de dados sistematizado contendo as informações das misturas do presente estudo. Os resultados de um primeiro incremento no banco de dados pode ser consultados em Gouveia et al. (2018). Para expandir o banco de dados atual, um passo a passo é sugerido a seguir para incorporação de futuras novas misturas ao banco de dados nacional:

a) Moldar os corpos de prova da mistura investigada com o Vv desejado e registrar o Vv;

b) Realizar ensaios de varredura de frequência em múltiplas temperaturas para a determinação do módulo dinâmico da mistura investigada;

c) Realizar o ensaio de varredura de frequência em múltiplas temperaturas para a determinação do módulo dinâmico do ligante asfáltico presente na mistura investigada. 0 ligante deve ser ensaiado depois de envelhecido no RTFOT. Caso a mistura contenha fresado, o ideal é ensaiar o ligante extraído após a moldagem de um corpo de prova da mistura correspondente - o ligante da mistura com fresado do presente estudo foi extraído e recuperado através das metodologias Soxhlet (DNIT 158/2011 - ME) e Abson (ASTM D 1856/1995), respectivamente;

d) Realizados os passos a, b e c, todos os parâmetros de entrada da equação proposta serão conhecidos e deverão ser organizados em planilha eletrônica;

e) Inserir os dados em um programa de análise de dados, a exemplo do LAB Fit (Silva e Silva, 2011), desenvolvido para auxiliar o tratamento dos dados experimentais e o refinamento da calibração das constantes do modelo de Mateos e Soares (2015);

f) Registrar, ainda, pelo menos as seguintes características: classificação ANP, teor, teor efetivo e curva mestra do ângulo de fase do ligante; agentes modificadores de ligante e/ou melhoradores de adesividade, se houver; distribuição granulométrica e TMN do agregado; Gmm, Gmb e método de dosagem da mistura. Tais informações podem contribuir para a realização de diferentes análises, e inclusive para a proposição de novos modelos.

\section{CONCLUSÕES}

Este trabalho avaliou o potencial de quatro modelos empíricos de previsão do módulo dinâmico de misturas asfálticas disponíveis na literatura internacional. Em geral, os modelos apresentaram correlações pelo menos razoáveis entre os módulos previstos e os obtidos em ensaios de 
misturas no laboratório. Entre os modelos investigados, os propostos por Sakhaeifar et al. (2015) e Mateos e Soares (2015) foram os que apresentaram correlações mais fortes entre estes dois grupos de dados. Além do forte potencial preditivo, a equação de Mateos e Soares (2015) é simples e baseada apenas no volume de vazios da mistura e no módulo dinâmico em cisalhamento do ligante. Assim, procedeu-se com a calibração de tal modelo a partir de um amplo banco de dados contendo 720 dados de módulos dinâmicos de misturas brasileiras.

Este esforço torna-se relevante na medida em que os modelos empíricos existentes na literatura foram calibrados considerando características de ligantes e misturas asfálticas dos seus países de origem. Como modelos empíricos são fortemente influenciados pelas características dos materiais consideradas na regressão dos dados experimentais, a definição de coeficientes específicos para misturas brasileiras é importante para possibilitar a obtenção de previsões ainda mais acuradas. Além disso, o modelo proposto e calibrado neste trabalho pode ter seu potencial preditivo aprimorado a partir do posterior refinamento da sua calibração considerando bancos de dados ainda mais extensos e representativos de diferentes regiões do país.

Espera-se que a versão calibrada do modelo preditivo adotado neste trabalho possa ser incorporada a versões futuras do novo método mecanístico-empírico de dimensionamento em desenvolvimento no país. Apesar de não ser recomendável a total substituição dos ensaios de laboratório por modelos empíricos como o calibrado neste trabalho, acredita-se que tais modelos podem ser úteis para a previsão dos módulos dinâmicos de misturas em situações em que os equipamentos de laboratório não estão disponíveis, em especial em projetos de baixo custo, a exemplo do que já ocorre na metodologia de dimensionamento americana. Adicionalmente, o uso de tais equações pode ser bastante atrativo para a otimização da fase de seleção de materiais, já que se pode avaliar de maneira eficiente e barata se determinada combinação de materiais resultará em misturas com módulos dinâmicos correspondentes aos previstos em anteprojetos.

\section{AGRADECIMENTOS}

Os autores agradecem os dados recebidos de diversas instituições do país, públicas e privadas, em especial UFC, UFSM, UFRGS e Grupo CCR, assim como o Projeto Rede Temática de Asfalto, capitaneado pela Petrobras. Agradecimentos também são devidos ao CNPq, pelas bolsas de produtividade do segundo, terceiro e quarto autores.

\section{REFÊNCIAS BIBLIOGRFICAS}

AASHTO (2011) AASHTO Designation T 342-11: Determining Dynamic Modulus of Hot-Mix Asphalt (HMA). American Association of State Highway and Transportation Officials, Washington, DC.

AASHTO (2012). AASHTO Designation T 315-12: Standard Method of Test for Determining the Rheological Properties of Asphalt Binder Using a Dynamic Shear Rheometer (DSR). American Association of State Highway and Transportation Officials, Washington, DC.

AL-KHATEEB, G. et al. (2006) A new simplistic model for dynamic modulus predictions of asphalt paving mixtures. Journal of the Association of Asphalt Paving Technologists - Proceedings of the Technical Sessions, v. 75, p. 1254-1293, Savannah, Georgia.

ALMEIDA JUNIOR, P. O. B. (2016) Comportamento mecânico de concretos asfálticos com diferentes granulometrias, ligantes e métodos de dosagem. 217 f. Dissertação (Mestrado em Engenharia Civil) - Universidade Federal de Santa Maria. Santa Maria.

ANDREI, D.; WITCZAK, M. W.; MIRZA, W. (1999) Development of revised predictive model for the dynamic (complex) modulus of asphalt mixtures. Development of the 2002 Guide for the Design of New and Rehabilitated Pavement Structures, NCHRP, Washington, DC.

AZARI, H. et al. (2007) Comparison of Simple Performance Test $\left|E^{*}\right|$ of Accelerated Loading Facility Mixtures and Prediction $\left|E^{*}\right|$ : Use of NCHRP 1-37A and Witczak's New Equations. Transportation Research Record: Journal of the Transportation Research Board, n. 1998, p. 1-9. DOI: 10.3141/1998-01.

BARI, J.; WITCZAK, M. W. (2006) Development of a new revised version of the Witczak E* predictive model for hot mix asphalt mixtures (with discussion). Journal of the Association of Asphalt Paving Technologists - Proceedings of the Technical Sessions v. 75, p. 381-423, Savannah, Georgia. 
BONNAURE, F. et al. (1977) New method of predicting the stiffness of asphalt paving mixtures. In: Association of Asphalt Paving Technologists - Proceedings of the Technical Sessions, v. 46, p. 64-104, San Antonio, Texas.

CEYLAN, H. et al. (2009) Accuracy of predictive models for dynamic modulus of hot-mix asphalt. Journal of Materials in Civil Engineering, v. 21, n. 6, p. 286-293. DOI: 10.1061/(asce)0899-1561(2009)21:6(286).

CHRISTENSEN JR, D. W.; PELLINEN, T.; BONAQUIST, R. F. (2003) Hirsch model for estimating the modulus of asphalt concrete. Journal of the Association of Asphalt Paving Technologists, v. 72, p. 97-121, Lexington, Kentucky.

CLYNE, T. R. et al. (2003) Dynamic and resilient modulus of Mn/DOT asphalt mixtures. MN/RC-2003-09, Final Report. Minnesota.

Departamento Nacional de Infraestrutura de Transportes (2010). Misturas betuminosas - Determinação do módulo de resiliência, DNIT-ME 135. 6p. Rio de Janeiro.

EL-BADAWY, S.; BAYOMY, F; AWED, A. (2012) Performance of MEPDG dynamic modulus predictive models for asphalt concrete mixtures: local calibration for Idaho. Journal of Materials in Civil Engineering, v. 24, n. 11, p. 1412-1421. DOI: 10.1061/(asce)mt.1943-5533.0000518.

EUROPEAN COMMITTEE FOR STANDARDIZATION (2012) EN 12697-26:2012 Bituminous mixtures: Test methods for hot mix asphalt - Part 26: Stiffness. Bruxelas.

FREIRE, R. A. (2015) Evaluation of the coarse aggregate influence in the fatigue damage using fine aggregate matrices with different maximum nominal sizes. 86 f. Dissertação (Mestrado em Engenharia de Transportes) - Universidade Federal do Ceará. Fortaleza.

GOUVEIA, B. C. S. (2016) Métodos de previsão e determinação de módulo dinâmico de misturas asfálticas aplicadas em rodovias brasileiras. 97 f. Dissertação (Mestrado em Engenharia de Transportes) - Universidade Federal do Ceará. Fortaleza.

GOUVEIA, B. C. S.; BASTOS, J. B. S.; SOARES, J. B. (2016) Contribuições quanto à resistência à deformação permanente de misturas asfálticas. In: $49^{\circ}$ Reunião Anual de Pavimentação, Brasília.

GOUVEIA, B. C. S. et al. (2018) Calibration and validation of empirical models to predict the dynamic modulus of Brazilian asphalt concrete mixtures. $9^{\text {th }}$ Annual Meeting Transportation Research Board, Washington.

KIM, Y. R.; KING, M.; MOMEN, M. (2005) Typical dynamic moduli for North Carolina asphalt concrete mixes. FHWA/NC/200503.

MATEOS, A.; SOARES, J. B. (2015) Validation of a dynamic modulus predictive equation on the basis of Spanish asphalt concrete mixtures. Materiales de Construcción, v. 65, n. 317, p. 047. DOI: 10.3989/mc.2015.01114

MATTOS, J. R. G. (2014) Monitoramento e análise do desempenho de pavimentos flexíveis da ampliação da rodovia BR290/RS: a implantação do Projeto Rede Temática de Asfalto no Rio Grande do Sul. 250 f. Tese (Doutorado em Engenharia Civil) - Universidade Federal do Rio Grande do Sul. Porto Alegre.

MOHAMMAD, L. N. et al. (2014) Characterization of Louisiana asphalt mixtures using simple performance tests and MEPDG. LTRC Report No. FHWA/LA, v. 11, p. 499.

OLIVEIRA, A. H. (2014) Avaliação do potencial antioxidante do líquido da castanha de caju (LCC) para materiais asfálticos através de diferentes métodos de envelhecimento. 140 f. Dissertação (Mestrado em Engenharia de Transportes) - Centro de Tecnologia, Universidade Federal do Ceará, Fortaleza.

ROBBINS, M. (2009) An investigation into dynamic modulus of hot-mix asphalt and its contributing factors. $174 \mathrm{f}$. Tese (Mestrado em Engenharia Civil) - Auburn University. Auburn.

ROBINETTE, C.; WILLIAMS, R. C. (2006) The effects of the testing history and preparation method on the Superpave simple performance test (with Discussion). Journal of the Association of Asphalt Paving Technologists - Proceedings of the Technical Sessions v. 75, p. 296-320, Savannah, Georgia.

SAKHAEIFAR, M. S.; KIM, Y. R.; KABIR, P. (2015) New predictive models for the dynamic modulus of hot mix asphalt. Construction and Building Materials, v. 76, p. 221-231. DOI: 10.1016/j.conbuildmat.2014.11.011

SCHWARTZ, C. W. (2005) Evaluation of the Witczak dynamic modulus prediction model. In: 84th Annual Meeting of the Transportation Research Board, Washington.

SPECHT, L. P. et al. (2017) Application of the theory of viscoelasticity to evaluate the resilient modulus test in asphalt mixes. Construction and Building Materials. Volume 149, Setembro, p. 648-658. DOI: 10.1016/j.conbuildmat.2017.05.037

UNDERWOOD, S.; ASHOURI, M.; KIM, Y. R. (2011) Effect on dynamic modulus measurement protocol on predicted pavement performance. Asphalt Paving Technology: Association of Asphalt Paving Technologists - Proceedings of the Technical Sessions. p. 65-98.

WITCZAK, M. W. et al. (2002) Simple performance test for Superpave mix design, NCHRP Report 465. Transportation Research Board, Washington. 